\title{
Evidence for High Spin Transition Metal Ion Induced Infrared Spectral Enhancement
}

James L. Gole ${ }^{\mathrm{a}}$, Sharka M. Prokes ${ }^{\mathrm{b}}$, Mark G. White, ${ }^{\mathrm{c}}$ Tsang-Hsiu Wang ${ }^{\mathrm{d}}$, Raluca Craciun ${ }^{\mathrm{d}}$, and David A. Dixon ${ }^{\mathrm{d}}$

a Dr. James L. Gole, Schools of Physics and Mechanical Engineering, Georgia Institute of Technology, 837 State Street, NW, Atlanta, GA 30332-0430 (USA) E-mail: jim.gole@physics.gatech.edu

${ }^{\mathrm{b}}$ Dr. S.M. Prokes, Code 6876, NRL, Washington, DC 20375 (USA) E-mail prokes@estd.nrl.navy.mil

${ }^{\mathrm{c}}$ Dr Mark G. White, Dave C. Swalm School of Chemical Engineering, James Worth Bagley College of Engineering, Box 9595, Mississippi State, MS 39762 E-Mail white@che.msstate.edu

${ }^{\mathrm{d}}$ Tsang-Hsiu Wang , Raluca Cranciun, and Dr. David A. Dixon, Department of Chemistry, University of Alabama, Tuscaloosa, Alabama 35487-0336

Supporting Information: Vibrational frequencies $\left(\mathrm{cm}^{-1}\right)$ for $\mathrm{H}_{2} \mathrm{O}, \mathrm{CH}_{3} \mathrm{NH}_{2}$, and $\mathrm{CH}_{3} \mathrm{NH}_{3}{ }^{+}$. 
Table SM1. Vibrational frequencies $\left(\mathrm{cm}^{-1}\right)$ for $\mathrm{H}_{2} \mathrm{O}, \mathrm{CH}_{3} \mathrm{NH}_{2}$, and $\mathrm{CH}_{3} \mathrm{NH}_{3}{ }^{+}$.

\begin{tabular}{|c|c|c|c|}
\hline \multirow[t]{2}{*}{ Mode } & Calc $^{\mathrm{a}}$ & $\begin{array}{l}\text { Calc } \\
\text { Scale }^{b}\end{array}$ & Expt $^{\mathrm{c}}$ \\
\hline & & \multicolumn{2}{|c|}{$\mathrm{H}_{2} \mathrm{O}\left(\mathrm{C}_{2} \mathrm{~V}\right)$} \\
\hline$b_{2}$ & 3952.0 & 3754 & 3756 \\
\hline$a_{1}$ & 3832.9 & 3641 & 3657 \\
\hline \multirow[t]{2}{*}{$a_{1}$} & 1630.2 & & 1595 \\
\hline & & \multicolumn{2}{|c|}{$\mathrm{CH}_{3} \mathrm{NH}_{2}$ (Cs) } \\
\hline a” & 3612.4 & 3432 & 3427 \\
\hline a' & 3530.9 & 3354 & 3361 \\
\hline a" & 3133.6 & 2977 & 2985 \\
\hline a’ & 3094.4 & 2940 & 2961 \\
\hline a' & 2998.0 & 2848 & 2820 \\
\hline a’ & 1665.0 & & 1623 \\
\hline a" & 1513.5 & & 1485 \\
\hline a' & 1495.9 & & 1473 \\
\hline a’ & 1454.5 & & 1430 \\
\hline a" & 1338.4 & & 1419 \\
\hline a' & 1162.0 & & 1130 \\
\hline a’ & 1060.2 & & 1044 \\
\hline a" & 969.8 & & 1195 \\
\hline a' & 845.5 & & 780 \\
\hline \multirow[t]{2}{*}{ a” } & 300.9 & & 268 \\
\hline & & \multicolumn{2}{|c|}{$\mathrm{CH}_{3} \mathrm{NH}_{3}{ }^{+}\left(\mathrm{C}_{3 \mathrm{v}}\right)$} \\
\hline e & 3521.5 & 3089 & \\
\hline$a_{1}$ & 3436.3 & 3264 & \\
\hline e & 3226.7 & 3065 & \\
\hline$a_{1}$ & 3114.9 & 2959 & \\
\hline e & 1677.8 & & \\
\hline$a_{1}$ & 1536.0 & & \\
\hline e & 1497.0 & & \\
\hline$a_{1}$ & 1465.0 & & \\
\hline e & 1272.5 & & \\
\hline$a_{1}$ & 936.2 & & \\
\hline e & 913.4 & & \\
\hline $\mathrm{a}_{2}$ & 296.8 & & \\
\hline
\end{tabular}

${ }^{\mathrm{a}}$ Calculated harmonic frequencies.

${ }^{\mathrm{b}}$ Calculated $\mathrm{C}-\mathrm{H}, \mathrm{N}-\mathrm{H}$, and $\mathrm{O}-\mathrm{H}$ stretches scaled by 0.95 .

${ }^{\mathrm{C}}$ Experimental frequencies from Shimanouchi, T. Tables of Molecular Vibrational

Frequencies Consolidated; National Bureau of Standards: Washington, D.C., 1972; Vol. I. 